\title{
Immunohistochemical expression of vascular endothelial growth factor and matrix metalloproteinase- 9 in radicular and residual radicular cysts
}

\author{
Patrícia Alvarez RUIZ1, Orlando Ayrton de TOLEDO², Cassiano Francisco Weege NONAKA ${ }^{3}$, Leão Pereira PINTO4, \\ Lélia Batista de SOUZA ${ }^{4}$
}

\footnotetext{
1- DDS, MSc, PhD, Professor, Department of Dentistry, Planalto Central Dental School, Brasília, DF, Brazil.

2- DDS, MSc, PhD, Professor, Department of Dentistry, University of Brasília, Brasília, DF, Brazil.

3 - DDS, MSc, Doctorate student, Department of Oral Pathology, Federal University of Rio Grande do Norte, Natal, RN, Brazil.

4 - DDS, MSc, PhD, Professor, Department of Oral Pathology, Federal University of Rio Grande do Norte, Natal, RN, Brazil.
}

Corresponding address: Lélia Batista de Souza - Departamento de Odontologia - Programa de Pós-Graduação em Patologia Oral - Av. Senador Salgado Filho, 1787 - Lagoa Nova - Natal - RN - Brasil - 59056-000 - Phone/ Fax: +55 84 3215-4138 - e-mail: cassiano_nonaka@yahoo.com.br

Received: March 09, 2009 - Modification: September 07, 2009 - Accepted: October 22, 2009

\section{ABSTRACT}

O bjective: This study assessed and compared the immunoexpression of vascular endothelial growth factor (VEGF) and matrix metalloproteinase-9 (MMP-9) in radicular cysts (RCs) and residual radicular cysts (RRCs), relating them to the angiogenic index and the intensity of the inflammatory infiltrate. Material and Methods: Twenty RCs and 10 RRCs were evaluated by immunohistochemistry using anti-VEGF and anti-MMP-9 antibodies. The angiogenic index was determined by microvessel count (MVC) using anti-von Willebrand factor antibody. Results: The expression of both VEGF and MMP-9 was higher in RCs than in RRCs. RCs and RRCs presented strong epithelial expression of VEGF, irrespective of the intensity of the inflammatory infiltrate. Lesions with strong expression of MMP-9 showed significantly higher number of immunopositive cells for VEGF $(p<0.05)$ and higher MVC $(p<0.05)$. Lesions with dense inflammatory infiltrate exhibited significantly higher MVC $(p<0.05)$ and higher number of immunopositive cells for VEGF $(p<0.05)$. There was a positive correlation between both MVC $(p<0.05)$ and the quantity of immunopositive cells for VEGF $(p<0.05)$, with intensity of the inflammatory infiltrate. In addition, it was observed a positive correlation between the number of immunopositive cells for VEGF and MVC $(p<0.05)$. Conclusions: VEGF and MMP-9 might play important roles in the angiogenesis in RCs and RRCs. In these lesions, the expression of these molecules and the MVC is closely related to the intensity of the inflammatory infiltrate. The expression of VEGF in the epithelial lining of RCs and RRCs might be important for the enlargement of these lesions.

Key words: Matrix metalloproteinase 9. Vascular endothelial growth factors. von Willebrand factor. Radicular cyst. Inflammation.

\section{INTRODUCTION}

Periapical lesions occur as a result of the immunological response to continuous antigenic stimulation from root canals ${ }^{36}$. As a consequence of inflammatory and immunological responses, the epithelial rests of Malassez are stimulated to proliferate, which may result in the development of radicular cysts $(R C s)^{18,24}$. If inadvertently left behind after the extraction of the involved tooth, RCs are named residual radicular cysts (RRCs) ${ }^{21}$. Although RCs and RRCs share similar histopathologic characteristics, these lesions may possess different biochemical properties, since the focus of stimulus is not present in RRCs ${ }^{24}$.

Recent studies suggest a role for vascular endothelial growth factor (VEGF) in the pathogenesis of $\mathrm{RCs}^{10,16,26}$ and RRCs ${ }^{26}$. VEGF is a potent proangiogenic cytokine which acts on the vasculature by inducing the proliferation, differentiation and migration of vascular endothelial 
cells ${ }^{4,6,32}$. Additionally, VEGF can induce microvascular permeability, leading to extravasation of plasma proteins, fluid accumulation and edema ${ }^{6,11,32}$. Thus, VEGF might be important for the development and evolution of RCs ${ }^{10,16}$ and $\mathrm{RRCs}^{26}$.

Angiogenesis is the process by which new blood vessels are produced by sprouting from preexisting vasculature $4,11,32$, and occurs by a series of sequential steps ${ }^{25,34}$. In response to angiogenic stimuli, endothelial cells degrade the basement membrane of microvessels by secreting proteolytic enzymes, including matrix metalloproteinases (MMPs), particularly MMPs-2 and 925,34. The cells then migrate through the degraded basement membrane and continue to break down the interstitial matrix as they move 25,34 . The endothelium aligns in a bipolar fashion to form a lumen and finally the newly formed hollow sprouts anastomosis with each other to form a capillary through which, blood flows ${ }^{25}$.

Results from studies in several pathologic processes have demonstrated the existence of an important relationship between VEGF and MMP-912,14,17. Hollborn, et al. ${ }^{12}$ (2007) verified that hypoxic expression of MMP-9 may stimulate the production and secretion of VEGF under pathologic conditions. In addition, Lee, et al. ${ }^{14}$ (2007) observed that intracerebral hemorrhage induced by VEGF is associated with increased expression of MMP-9.

Studies investigating the expression of MMP-91,5 and VEGF ${ }^{10,16,26}$ in periapical lesions are scarce. To the best of our knowledge, there are no studies analyzing the expression of both VEGF and MMP-9 in RCs and RRCs. Therefore, the aim of this study was to assess and compare the immunoexpression of these molecules in RCs and RRCs, relating them to the angiogenic index and the intensity of the inflammatory infiltrate.

\section{MATERIAL AND METHODS}

Thirty tissue specimens, 20 RCs and 10 RRCs, from the files of the Department of Oral Pathology of the Federal University of Rio Grande do Norte (UFRN), Brazil, were randomly selected for this study. All RCs were obtained from teeth without endodontic treatment. In addition, all lesions presented unequivocal cystic cavity lined by odontogenic epithelium. The cases were not matched for age, sex, or anatomic location. Serial 5 - $\mu \mathrm{m}$-thick and 3- $\mu \mathrm{m}$-thick sections were taken from tissue blocks and processed for morphological and immunohistochemical studies, respectively. The study was approved by the Research Ethics Committee of the University of Brasília, Brazil.

\section{Morphological study}

Tissue sections were stained with hematoxylin and eosin technique. The intensity of the inflammatory infiltrate was evaluated according to the method proposed by Tsai, et al. ${ }^{33}$ (2004). Each specimen was graded at $\times 200$ magnification as: grade $I$, inflammatory cells less than $1 / 3$ per field; grade II, inflammatory cells between $1 / 3$ and $2 / 3$ per field; and grade III, inflammatory cells higher than 2/3 per field. Grading of each specimen was recorded on the average inflammatory condition in three consecutive microscopic fields, starting from the inner portion of the specimen and proceeding deeper into connective tissue. Thickness of the epithelial lining was defined as atrophic (2-10 cell layers) or hyperplastic (>10 cell layers), according to Moreira, et al. ${ }^{22}$ (2000).

\section{Immunohistochemical methods}

Tissue sections were deparaffinized and immersed in methanol with $0.3 \%$ hydrogen peroxide to block endogenous peroxidase activity. The tissue sections were then washed in phosphate-bufferedsaline (PBS). Antigen retrieval for antibody antiVEGF (C-1 clone; Santa Cruz Biotechnology, Santa Cruz, CA, USA) was performed in oven (Trypsin $\mathrm{pH} 7.9,60 \mathrm{~min})$. Antigen retrieval for antibodies anti-MMP-9 (2C3 clone; Novocastra, Newcastle, England, UK) and anti-vWF (F8/86 clone; Dako, Glostrup, Copenhagen, DEN) was performed in steamer (citrate pH 6.0, $30 \mathrm{~min}$ ). In sequence, the tissue sections were incubated with primary mouse antibodies anti-VEGF (dilution 1:400, overnight), anti-MMP-9 (dilution 1:20, overnight), and antivWF (dilution 1:50, $60 \mathrm{~min}$ ). The tissue sections were then washed twice in PBS and treated with streptavidin-biotin-peroxidase complex (Dako) at room temperature in order to bind the primary antibodies. Peroxidase activity was visualized by immersing tissue sections in diaminobenzidine (D5637; Sigma Chemical, St. Louis, MO, USA), resulting in a brown reaction product. Finally, tissue sections were counterstained with Mayer's hematoxylin and coverslipped. Positive controls were sections of normal human kidney for VEGF and $V W F$, and sections of periapical granuloma for MMP-9. As negative controls, samples were treated as above, except that the primary antibody was replaced by a solution of bovine serum albumin (BSA) in PBS.

\section{Immunostaining assessment and statistical analysis}

Immunoexpression of VEGF was evaluated both in the connective tissue and in the epithelial lining of RCs and RRCs. In the connective tissue, a quantitative assessment of the immunopositive cells was performed, irrespective of the color intensity, according to the method proposed by Freitas, et al. ${ }^{8}$ (2005). Tissue sections were examined under light microscopy at $\times 100$ magnification in order 
to identify five fields with the largest number of immunostained cells. Using $\times 400$ magnification, the counting of the immunopositive cells was performed in each one of these fields.

The immunoexpression of VEGF in the epithelial lining was semi-quantitatively evaluated at $\times 100$ magnification. Performing an adaptation of the method proposed by Leonardi, et al. ${ }^{16}$ (2003), the epithelial immunoexpression of VEGF was classified according to the following scores: 0 - no staining; 1 - weak, staining in $11-25 \%$ of cells; score 2 moderate, staining in $26-75 \%$ of cells; 3 - strong, staining in more than $76 \%$ of cells.

The immunoexpression of MMP-9 was semiquantitatively evaluated at $\times 200$ magnification. The method proposed by Franchi, et al.7 (2002) was adapted. Thus, the expression of MMP-9 was assessed in endothelial cells of vessels with conspicuous lumen and classified according to the scores: 0 - no staining; 1 - weak, staining in less than $10 \%$ of vessels; 2 - moderate, staining in 11 $50 \%$ of vessels; 3 - strong, staining in more than $51 \%$ of vessels.

Angiogenic index was determined based on the number of vessels immunoreactive to anti-vWF antibody. Adopting the methodology utilized by
Freitas, et al. ${ }^{8}$ (2005), a microvessel count (MVC) was performed. Tissue sections were examined under light microscopy at $\times 40$ magnification and five areas showing the highest vascularization were identified subjectively. In these areas, vessels were counted at $\times 200$ magnification.

The results obtained were submitted to statistical analysis. Computations were made using the Statistical Package for the Social Sciences (SPSS 13.0). To analyze the immunoexpression of VEGF in the epithelial lining and the expression of MMP9 in blood vessels, Mann-Whitney nonparametric test was performed. Comparison of the number of cells immunoreactive for VEGF in the connective tissue and MVC was performed by the KruskalWallis and Mann-Whitney nonparametric tests. Finally, Spearman's correlation test was performed in order to verify possible correlations between the variables analyzed. For all tests, a $p$ value $<0.05$ was considered to be significant.

\section{RESULTS}

\section{Morphological analysis}

In RCs, 14 cases (70\%) showed inflammatory infiltrate grade III, 5 cases (25\%) presented

Table 1- Parameters used for the calculation of the Mann-Whitney $U$ test for the evaluation of the expression of vascular endothelial growth factor (VEGF), von Willebrand factor (VWF), and matrix metalloproteinase-9 (MMP-9) according to the type of lesion

\begin{tabular}{lllccccc}
\hline Marker & Lesion & $\mathbf{n}$ & Median & $\mathbf{Q}_{\mathbf{2 5}}-\mathbf{Q}_{\mathbf{7 5}}$ & Mean of the ranks & $\mathbf{U}$ & $\mathbf{p}$ \\
\hline \multirow{2}{*}{ VEGF } & RC & 20 & 590.50 & $505.75-688.75$ & 17.20 & 66.00 & 0.135 \\
& RRC & 10 & 460.00 & $256.00-618.50$ & 12.10 & & \\
VWF & RC & 20 & 233.00 & $199.00-287.50$ & 17.00 & 70.00 & 0.187 \\
& RRC & 10 & 199.50 & $145.50-277.75$ & 12.50 & & \\
MMP-9 & RC & 20 & 3.0 & $2.0-3.0$ & 17.30 & 64.00 & 0.067 \\
& RRC & 10 & 1.0 & $1.0-3.0$ & 11.90 & & \\
\hline
\end{tabular}

Legend: RC - Radicular cyst; RRC - Residual radicular cyst

Table 2- Parameters used for the calculation of the Kruskal-Wallis (KW) test for the evaluation of the expression of vascular endothelial growth factor (VEGF), von Willebrand factor (VWF), and matrix metalloproteinase-9 (MMP-9) according to the grade of the inflammatory infiltrate

\begin{tabular}{|c|c|c|c|c|c|c|c|}
\hline Marker & Inflammatory infiltrate & $\mathbf{n}$ & Median & $Q_{25}-Q_{75}$ & Mean of the ranks & $\mathrm{KW}$ & $\mathbf{p}$ \\
\hline \multirow[t]{3}{*}{ VEGF } & Grade la & 5 & 373.00 & $242.00-547.50$ & 8.20 & 6.660 & 0.036 \\
\hline & Grade Ila & 8 & 412.00 & $245.00-636.50$ & 12.88 & & \\
\hline & Grade IIIb & 17 & 611.00 & $549.50-682.50$ & 18.88 & & \\
\hline \multirow[t]{3}{*}{ vWF } & Grade la & 5 & 173.00 & $154.00-240.00$ & 9.80 & 9.208 & 0.010 \\
\hline & Grade Ila & 8 & 191.00 & $139.75-220.75$ & 10.00 & & \\
\hline & Grade IIIb & 17 & 257.00 & $218.50-342.50$ & 19.76 & & \\
\hline \multirow[t]{3}{*}{ MMP-9 } & Grade I & 5 & 1.0 & $1.0-2.0$ & 8.70 & 5.492 & 0.064 \\
\hline & Grade II & 8 & 2.50 & $1.25-3.0$ & 15.00 & & \\
\hline & Grade III & 17 & 3.0 & $2.0-3.0$ & 17.74 & & \\
\hline
\end{tabular}

*Different letters represent statistically significant difference 

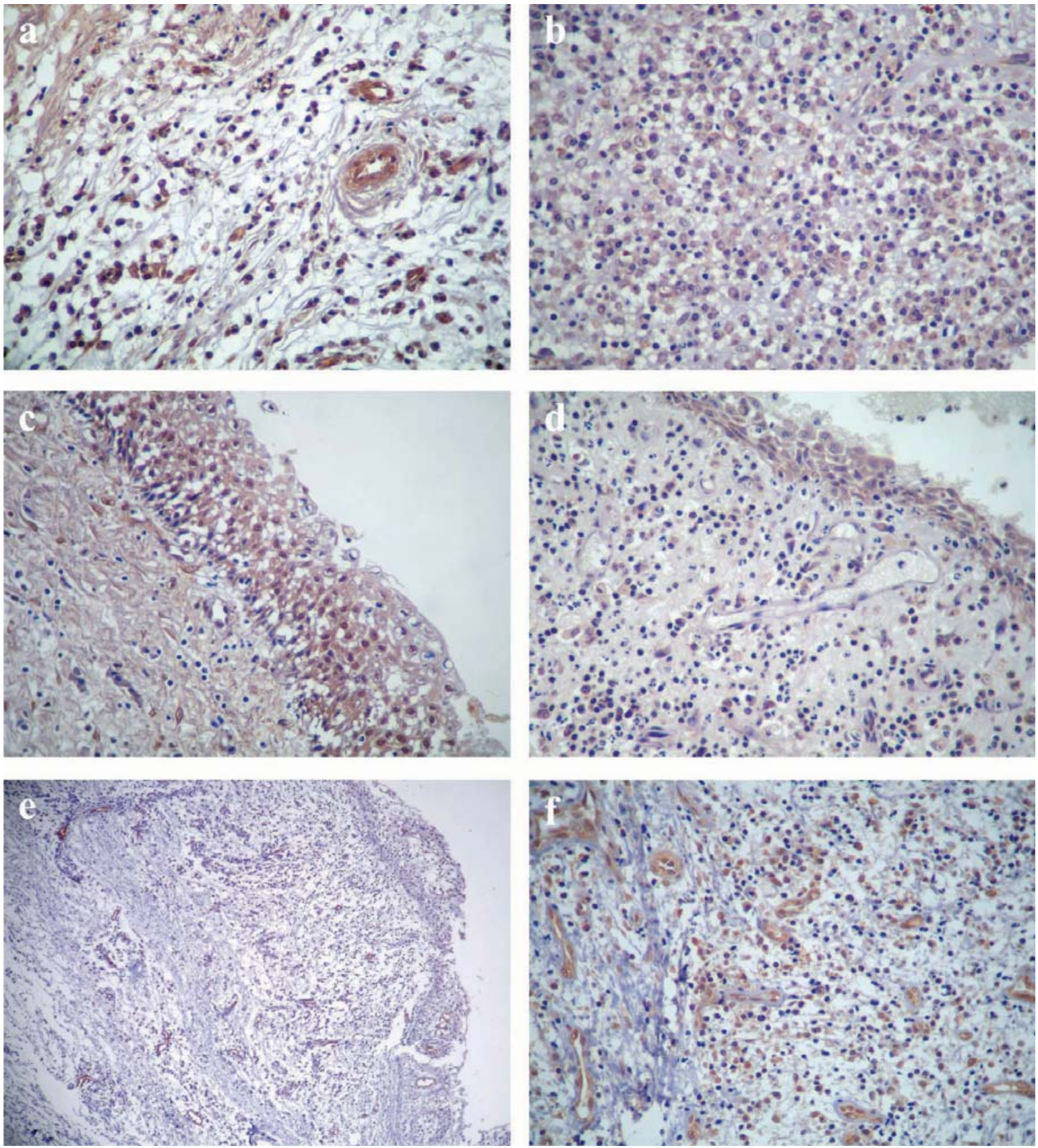

Figure 1- (a) Immunoexpression of vascular endothelial growth factor (VEGF) in residual radicular cyst showing inflammatory infiltrate grade I (SABC method, original magnification $\times 400$ ). (b) Immunoexpression of VEGF in radicular cyst showing inflammatory infiltrate grade III (SABC method, original magnification $\times 400$ ). (c) Strong epithelial expression of VEGF in residual radicular cyst with hyperplastic epithelial lining (SABC method, original magnification $\times 400)$. (d) Strong epithelial expression of VEGF in radicular cyst with atrophic epithelial lining (SABC method, original magnification $\times 400)$. (e) Vessels labeled by anti-vWF antibody in a specimen of residual radicular cyst (SABC method, original magnification $\times 100)$. ( $f$ ) Endothelial cells showing strong immunoreactivity for MMP-9 in a specimen of radicular cyst (SABC method, original magnification $\times 400$ )

inflammatory infiltrate grade II, and only 1 case (5\%) revealed inflammatory infiltrate grade $\mathrm{I}$. In RRCs, 4 cases (40\%) showed inflammatory infiltrate grade I, 3 cases (30\%) exhibited inflammatory infiltrate grade II and 3 cases (30\%) revealed inflammatory infiltrate grade III.

Analysis of the epithelial thickness in RCs revealed a hyperplastic epithelium in 14 cases $(70 \%)$ and an atrophic epithelium in 6 cases (30\%). In RRCs, 6 cases $(60 \%)$ presented an atrophic epithelium and 4 cases (40\%) showed a hyperplastic epithelium. 
Table 3- Parameters used for the calculation of the Mann-Whitney $U$ test for the evaluation of the epithelial expression of vascular endothelial growth factor (VEGF) according to the type of lesion and epithelial thickness

\begin{tabular}{lllccccc}
\hline Variable & Group & $\mathbf{n}$ & Median & $\mathbf{Q}_{25}-\mathbf{Q}_{\mathbf{7 5}}$ & Mean of the ranks & $\mathbf{U}$ & $\mathbf{p}$ \\
\hline \multirow{2}{*}{ Type of lesion } & & & & & & & \\
\multirow{2}{*}{ Epithelial thickness } & 20 & 3.0 & $2.0-3.0$ & 14.60 & 82.00 & 0.360 \\
& RRC & 10 & 3.0 & $2.0-3.0$ & 17.30 & & \\
& Atrophic & 12 & 3.0 & $2.0-3.0$ & 15.83 & 104.00 & 0.845 \\
\hline
\end{tabular}

Legend: RC - Radicular cyst; RRC - Residual radicular cyst

Table 4- Parameters used for the calculation of the Mann-Whitney $U$ test for the evaluation of the expression of vascular endothelial growth factor (VEGF) and von Willebrand factor (VWF) according to the expression of matrix metalloproteinase-9 (MMP-9)

\begin{tabular}{lccccccc}
\hline Marker & Expression of MMP-9 & $\mathbf{n}$ & Median & $\mathbf{Q}_{\mathbf{2 5}}-\mathbf{Q}_{\mathbf{7 5}}$ & Mean of the ranks & $\mathbf{U}$ & $\mathbf{p}$ \\
\hline \multirow{2}{*}{ VEGF } & Weak/ Moderate & 12 & 335.50 & $244.00-555.25$ & 9.25 & 33.00 & 0.001 \\
& Strong & 18 & 618.50 & $546.25-671.25$ & 19.67 & & \\
\multirow{2}{*}{ vWF } & Weak/ Moderate & 12 & 177.00 & $153.00-241.75$ & 9.67 & 38.00 & 0.003 \\
& Strong & 18 & 254.50 & $213.25-351.25$ & 19.39 & & \\
\hline
\end{tabular}

\section{Immunohistochemical analysis}

The mean number of cells immunopositive for VEGF was 565.05 and 443.90 in RCs and RRCs, respectively $(p=0.135)$ (Table 1$)$. Grouping all lesions according to the intensity of the inflammatory infiltrate, the mean number of immunopositive cells for VEGF was 390.40 in lesions with inflammatory infiltrate grade I (Figure 1a), 450.00 in lesions with inflammatory infiltrate grade II, and 599.29 in lesions with inflammatory infiltrate grade III $(p=0.036)$ (Table 2) (Figure 1b).

Analysis of the epithelial expression of VEGF disclosed a predominance of strong immunoreactivity (score 3 ) for both RCs and RRCs $(p=0.360)$ (Table $3)$, irrespective of the intensity of the inflammatory infiltrate. Regarding the expression of VEGF in relation to the epithelial thickness, there was a predominance of strong immunoreactivity (score 3 ) for both cysts with hyperplastic epithelium (Figure $1 c)$ and cysts with atrophic epithelium $(p=0.845)$ (Table 3) (Figure 1d).

The mean number of blood vessels determined by MVC was 250.85 in RCs (Figure 1e), and 217.00 in RRCs $(p=0.187)$ (Table 1). Grouping all lesions according to the intensity of the inflammatory infiltrate, the mean number of blood vessels was 192.20 in lesions with inflammatory infiltrate grade I, 198.00 in lesions with inflammatory infiltrate grade II, and 273.06 in lesions with inflammatory infiltrate grade III $(p=0.010)$ (Table 2).

Analysis of the immunoexpression of MMP-9 in the endothelial cells disclosed a predominance of strong immunoreactivity (score 3) for RCs (Figure 1f), and a predominance of weak immunoreactivity (score 1) for RRCs $(p=0.067)$ (Table 1). There was a predominance of weak expression (score 1) of MMP-9 for lesions with inflammatory infiltrate grade I, whereas lesions with inflammatory infiltrate grade II revealed a predominance of moderate to strong expression (score 2-3) of this protease (Table 2). Lesions with inflammatory infiltrate grade III revealed a predominance of strong expression (score 3 ) of MMP-9 (Table 2). There was no significant difference in the expression of MMP-9 in relation to the intensity of the inflammatory infiltrate $(p=0.064)$ (Table 2$)$.

In order to evaluate differences in the MVC and in the number of immunopositive cells for VEGF according to the expression of MMP-9 in endothelial cells, cases showing weak and moderate expression of MMP-9 were grouped and compared with cases presenting high expression of MMP9. Lesions showing high expression of MMP-9 in endothelial cells exhibited higher number of both immunopositive cells for VEGF $(p=0.001)$ (Table 4) and blood vessels $(p=0.003)$ (Table 4$)$.

Spearman's correlation test disclosed a positive correlation between the number of immunopositive cells for VEGF $(r=0.477 ; p=0.008)$ as well as the angiogenic index $(r=0.545 ; p=0.002)$ and intensity of the inflammatory infiltrate. A positive correlation between the number of immunopositive cells for VEGF and the angiogenic index ( $r=0.519$; $p=0.003$ ) was also observed. Finally, there was a positive correlation between both number of immunopositive cells for VEGF $(r=0.540 ; p=0.002)$ and angiogenic index $(r=0.539 ; \mathrm{p}=0.002)$, with expression of MMP-9 in endothelial cells. 


\section{DISCUSSION}

The importance of angiogenesis in the development and progression of periapical lesions has been highlighted in a number of studies ${ }^{10,16,26}$. VEGF, a dimeric glycoprotein with a selective mitogenic effect on vascular endothelial cells ${ }^{4,6,11,32}$, plays several pivotal functions in angiogenesis ${ }^{4,11}$. This proangiogenic cytokine is capable of inducing microvascular permeability, leading to extravasation of plasma proteins and a predictable sequence of proangiogenic stromal changes ${ }^{16}$. Due to these functions, VEGF has been implicated as an important factor in granulation tissue development ${ }^{4,11}$, and cyst enlargement ${ }^{30,35}$.

In the present study, VEGF expression was observed both in the connective tissue and in the epithelial lining of RCs and RRCs, corroborating previous reports ${ }^{10,16,26}$. In line with other studies ${ }^{10,26}$, we verified that lesions with a dense inflammatory infiltrate (grade III) presented both a large number of immunopositive cells for VEGF $(p<0.05)$ and a high MVC $(p<0.05)$. Moreover, there was a positive correlation between both number of immunopositive cells for VEGF $(p<0.05)$ and MVC $(p<0.05)$, with the intensity of the inflammatory infiltrate. These findings emphasize the importance of inflammatory cells on VEGF expression ${ }^{10,26}$ and, consequently, in angiogenesis itself ${ }^{27}$. The low level of inflammation in RRCs, probably due to a reduced antigenic stimulation ${ }^{24}$, could be responsible for the Iow expression of VEGF, and consequently low MVC, in these lesions.

Graziani, et al. ${ }^{10}$ (2006) verified a positive correlation between the expression of VEGF and the microvessel density. Despite using a different method for quantification of vessels, we could also find a statistically significant correlation between the number of immunopositive cells for VEGF and the quantity of vessels immunoreactive for anti-vWF antibody. Such findings corroborate the mitogenic effect of VEGF on vascular endothelial cells described in the literature $4,6,11,32$.

The epithelium status of the RCs has been suggested as a reliable histological parameter of biological activity and/or inactivity of cystic growth $^{22}$. On lesions with atrophic epithelium, despite the presence of antigens and enzymes able to induce immunological responses, epithelium proliferation and bone resorption, cyst enlargement does not occur ${ }^{22}$. Immunosuppressor effectors ${ }^{22}$ or apoptotic events ${ }^{19}$ are supposed to operate in such phases, regulating cyst enlargement. In spite of those reports, our results revealed a predominance of strong epithelial immunoreactivity (score 3) for VEGF in RCs, irrespective of the epithelial lining thickness. Therefore, the epithelial expression of VEGF in RCs might constitute an additional mechanism for the enlargement of these lesions, maintaining the stimulus for angiogenesis and vascular permeability ${ }^{16,26}$. Results from studies on brain tumor cysts ${ }^{30,35}$ suggest that VEGF might enhance accumulation of cyst fluid altogether with an increase in oxygen supply boosting the development of the cyst ${ }^{35}$.

The RRCs have been described as lesions with lower epithelial proliferative potential, in comparison to $\mathrm{RCs}^{31}$. In the present study, there was a predominance of strong epithelial immunoreactivity (score 3) for VEGF in RRCs, irrespective of the intensity of the inflammatory infiltrate or the thickness of the epithelial lining. Thus, in spite of the low proliferative potential reported in the literature ${ }^{31}$, the expression of VEGF by the epithelial lining of RRCs might contribute with cyst enlargement. Accordingly, Muglali, et al. ${ }^{24}$ (2008) verified the presence of interleukin-1a, monocyte chemotactic protein 1 , and regulated upon activation normal T-cell expressed and secreted (RANTES) in cystic fluids of RRCs, suggesting the existence of a potential for expansion in these lesions.

MMP-9 is a proteolytic enzyme that can degrade type IV collagen and laminin, major components of basement membranes ${ }^{3}$, and thereby allows proteolysis-associated migration of endothelial cells ${ }^{12,14}$. In addition to this proteolytic activity, MMP-9 has been involved in activation of growth factors and cytokines ${ }^{12,23,34}$, recruitment of endothelial progenitor cells ${ }^{12,34}$, and generation of anti-angiogenic factors, such as angiostatin and tumstatin $^{3,34}$. Thus, MMP-9 has been identified as an essential molecule for angiogenesis both in pathologic and physiologic conditions ${ }^{25,34}$.

Studies about the expression of MMP-9 in periapical lesions are scarce ${ }^{1,5}$ and their results suggest that MMP-9 might play a role in the extracellular matrix destruction associated with the development/progression of these lesions ${ }^{5}$. Moreover, according to Belmar, et al. ${ }^{1}$ (2008), MMP9 could represent a useful marker in monitoring chronic periapical periodontitis in gingival crevicular fluid. To our knowledge, there are no studies evaluating the participation of MMP-9 in the angiogenesis on RCs and RRCs.

A high percentage of RCs evaluated in this study showed strong expression of MMP-9 in the endothelial cells, whereas the RRCs exhibited a tendency for a weaker expression of this protease. In addition, despite the absence of statistical significance $(p>0.05)$, most lesions with inflammatory infiltrate grade I showed weak expression of MMP-9, whereas a high proportion of lesions with inflammatory infiltrate grade III revealed strong expression of this protease.

Many inflammatory cells, including neutrophils 
and macrophages, are able to produce a series of angiogenic factors, such as platelet derived growth factor (PDGF), fibroblastic growth factor-2 (FGF-2), tumor necrosis factor-alpha (TNF- $\alpha$ ) and VEGF27. TNF-a is known to stimulate secretion of MMP-9 in microvascular endothelial cells ${ }^{20}$. In addition, studies have reported a positive correlation between VEGF and MMP-9 ${ }^{12,17}$. Coherently, Lee, et al. ${ }^{15}$ (2006) analyzed the expression of VEGF and MMP-9 in asthma and verified that inhibition of VEGF receptor down-regulates the expression of MMP-9.

Jurisic, et al. ${ }^{13}$ (2008) showed a positive correlation between the concentration of TNF- $\alpha$ and the number of inflammatory cells in RCs. In agreement with the present study, Nonaka, et al. ${ }^{26}$ (2008) observed a lower number of immunopositive cells for VEGF in periapical lesions with few inflammatory infiltrate. Therefore, the expression of MMP-9 in endothelial cells of RCs and RRCs might be related to the intensity of the inflammatory infiltrate in these lesions, a fact that could also explain the tendency for a weaker expression of this protease in RRCs.

In addition to the synthesis of proangiogenic factors, inflammatory cells might also contribute with angiogenesis through secretion of MMP-9. Studies about carcinogenesis in mouse models verified that the mobilization of extracellular VEGF from matrix stores constitutes an important event during tumor angiogenesis ${ }^{9,28}$. This mobilization was dependent on MMP-9 activity from inflammatory cells, particularly macrophages and neutrophils ${ }^{9,28}$. According to Mott and Werb ${ }^{23}$ (2004), although these are tumor models, it is highly likely that similar mechanisms for the release of VEGF occur in non-pathological conditions. Data obtained from the study of Carneiro, et al. ${ }^{5}$ (2009) suggests the participation of several inflammatory cells, mainly macrophages, in the MMP-9 expression in apical periodontitis lesions. Thus, it could be hypothesized that secretion of MMP-9 by inflammatory cells in RCs and RRCs might contribute with angiogenesis through mobilization of VEGF from the matrix stores, making it more available to VEGF receptors. Accordingly, VEGF has been recognized as a bioactive substrate for MMP-92,29.

In the present study, lesions with strong expression of MMP-9 in endothelial cells showed both a larger number of immunopositive cells for VEGF $(p<0.05)$ and a higher MVC $(p<0.05)$ It could also be observed a positive correlation between the expression of MMP-9 and both the number of immunopositive cells for VEGF $(p<0.05)$ and the angiogenic index $(p<0.05)$. These results agree with the positive correlation between VEGF and MMP-9 reported in the literature ${ }^{12,17}$. Hollborn, et al. ${ }^{12}$ (2007) observed that MMP-9 causes the upregulation of VEGF expression in retinal pigmented cells. In face of their findings, the authors suggested that MMP-9 also may facilitate pathologic neovascularization through stimulation of the production of angiogenic factors.

According to $\mathrm{Li}$, et al. ${ }^{17}$ (2006), angiogenesis is a process that not only needs several vascular growth factors, but also requires degradation of vascular basement membrane and remodeling of the extracellular matrix to enable endothelial cells to migrate into surrounding tissues. Thus, the present results suggest that MMP-9 might be an important molecule for angiogenesis in RCs and RRCs, probably promoting degradation of components of vascular basement membrane ${ }^{3}$, and thereby allowing proteolysis-associated migration of endothelial cells ${ }^{12,14}$.

\section{CONCLUSIONS}

In conclusion, our results suggest an important role for VEGF and MMP-9 in the angiogenesis on RCs and RRCs. In these lesions, the expression of these molecules and the MVC is closely related to the intensity of the inflammatory infiltrate. The expression of VEGF in the epithelial lining of RCs and RRCs might be important for the enlargement of these lesions.

\section{ACKNOWLEDGEMENTS}

We thank professor Kenio Costa Lima for his assistance with the statistical analysis.

\section{REFERENCES}

1- Belmar MJ, Pabst C, Martínez B, Hernández M. Gelatinolytic activity in gingival crevicular fluid from teeth with periapical lesions. Oral Surg Oral Med Oral Pathol Oral Radiol Endod. 2008;105:801-6.

2- Bergers G, Brekken R, McMahon G, Vu TH, Itoh T, Tamaki K, et al. Matrix metalloproteinase- 9 triggers the angiogenic switch during carcinogenesis. Nat Cell Biol. 2000;2:737-44.

3- Björklund M, Koivunen E. Gelatinase-mediated migration and invasion of cancer cells. Biochim Biophys Acta. 2005;1755:37-69. 4- Byrne AM, Bouchier-Hayes DJ, Harmey JH. Angiogenic and cell survival functions of vascular endothelial growth factor (VEGF). J Cell Mol Med. 2005;9:777-94.

5- Carneiro E, Menezes R, Garlet GP, Garcia RB, Bramante CM, Figueira $\mathrm{R}$, et al. Expression analysis of matrix metalloproteinase- 9 in epithelialized and nonepithelialized apical periodontitis lesions. Oral Surg Oral Med Oral Pathol Oral Radiol Endod 2009;107:12732.

6- Ferrara N, Davis-Smyth T. The biology of vascular endothelial growth factor. Endocr Rev. 1997;18:4-25.

7- Franchi A, Santucci M, Masini E, Sardi I, Paglierani M, Gallo O. Expression of matrix metalloproteinase 1, matrix metalloproteinase 2 , and matrix metalloproteinase 9 in carcinoma of the head and neck. Cancer. 2002;95:1902-10.

8- Freitas TM, Miguel MC, Silveira EJ, Freitas RA, Galvão HC. Assessment of angiogenic markers in oral hemangiomas and pyogenic granulomas. Exp Mol Pathol. 2005;79:79-85. 
9- Giraudo E, Inoue M, Hanahan D. An amino-biphosphonate targets MMP-9-expressing macrophages and angiogenesis to impair cervical carcinogenesis. J Clin Invest. 2004;114:623-33. 10- Graziani F, Vano M, Viacava P, Itro A, Tartaro G, Gabriele M. Microvessel density and vascular endothelial growth factor (VEGF) expression in human radicular cysts. Am J Dent. 2006;19:11-4. 11- Hoeben A, Landuyt B, Highley MS, Wildiers $H$, Van Oosterom AT, De Bruijn EA. Vascular endothelial growth factor and angiogenesis. Pharmacol Rev. 2004.56:549-80.

12- Hollborn M, Stathopoulos C, Steffen A, Wiedemann P, Kohen $\mathrm{L}$, Bringmann A. Positive feedback regulation between MMP9 and VEGF in human RPE cells. Invest Ophthalmol Vis Sci. 2007;48:4360-7.

13- Jurisic V, Terzic T, Colic S, Jurisic M. The concentration of TNFalpha correlates with number of inflammatory cells and degree of vascularization in radicular cysts. Oral Dis. 2008;14:600-5.

14- Lee CZ, Xue Z, Zhu Y, Yang GY, Young WL. Matrix metalloproteinase- 9 inhibition attenuates vascular endothelial growth factor-induced intracerebral hemorrhage. Stroke. 2007;38:2563-8.

15- Lee KS, Min KH, Kim SR, Park SJ, Park HS, Jin GY, et al. Vascular endothelial growth factor modulates matrix metalloproteinase- 9 expression in asthma. Am J Respir Crit Care Med. 2006;174:161-70.

16- Leonardi R, Caltabiano M, Pagano M, Pezzuto V, Loreto C, Palestro $G$. Detection of vascular endothelial growth factor/vascular permeability factor in periapical lesions. J Endod. 2003;29:180-3. 17- Li T, Li YG, Pu DM. Matrix metalloproteinase-2 and -9 expression correlated with angiogenesis in human adenomyosis. Gynecol Obstet Invest. 2006;62:229-35.

18- Lia RC, Garcia JM, Sousa-Neto MD, Saquy PC, Marins RH, Zucollotto WG. Clinical, radiographic and histological evaluation of chronic periapical inflammatory lesions. J Appl Oral Sci. 2004; $12: 117-20$

19- Loyola AM, Cardoso SV, Lisa GS, Oliveira LJ, Mesquita RA, Carmo MA, et al. Apoptosis in epithelial cells of apical radicular cysts. Int Endod J. 2005;38:465-9.

20- Majka S, McGuire PG, Das A. Regulation of matrix metalloproteinase expression by tumor necrosis factor in a murine model of retinal neovascularization. Invest Ophthalmol Vis Sci. 2002:43:260-6.

21- Moldauer I, Velez I, Kuttler S. Upregulation of basic fibroblast growth factor in human periapical lesions. J Endod. 2006;32:40811.

22- Moreira PR, Santos DF, Martins RD, Gomez RS. CD57+ in radicular cyst. Int Endod J. 2000;33:99-102.
23- Mott JD, Werb Z. Regulation of matrix biology by matrix metalloproteinases. Curr Opin Cell Biol. 2004;16:558-64. 24- Muglali M, Komerik N, Bulut E, Yarim GF, Celebi N, Sumer M. Cytokine and chemokine levels in radicular and residual cysts fluids. J Oral Pathol Med. 2008;37:185-9.

25- Nguyen M, Arkell J, Jackson CJ. Human endothelial gelatinases and angiogenesis. Int J Biochem Cell Biol. 2001;33:960-70.

26- Nonaka CF, Maia AP, Nascimento GJ, Almeida Freitas R, Batista de Souza L, Galvão HC. Immunoexpression of vascular endothelial growth factor in periapical granulomas, radicular cysts, and residual radicular cysts. Oral Surg Oral Med Oral Pathol Oral Radiol Endod. 2008;106:896-902.

27- Noonan DM, De Lerma Barbaro A, Vannini N, Mortara L, Albini A. Inflammation, inflammatory cells and angiogenesis: decisions and indecisions. Cancer Metastasis Rev. 2008;27:31-40.

28- Nozawa $\mathrm{H}$, Chiu C, Hanahan D. Infiltrating neutrophils mediate the initial angiogenic switch in a mouse model of multistage carcinogenesis. Proc Natl Acad Sci USA. 2006;103:12493-8.

29- Overall CM. Molecular determinants of metalloproteinase substrate specificity: matrix metalloproteinase substrate binding domains, modules, and exosites. Mol Biotechnol. 2002;22:51-86. 30- Stockhammer G, Obwegeser A, Kostron H, Schumacher $P$, Muigg A, Felber S, et al. Vascular endothelial growth factor (VEGF) is elevated in brain tumor cysts and correlates with tumor progression. Acta Neuropathol. 2000;100:101-5.

31- Suzuki T, Kumamoto H, Ooya K, Motegi K. Immunohistochemical analysis of CD1a-labeled Langerhans cells in human dental periapical inflammatory lesions - correlation with inflammatory cells and epithelial cells. Oral Dis. 2001;7:336-43.

32- Takahashi $H$, Shibuya $M$. The vascular endothelial growth factor (VEGF)/ VEGF receptor system and its role under physiological and pathological conditions. Clin Sci (Lond). 2005;109:227-41. 33- Tsai CH, Weng SF, Yang LC, Huang FM, Chen YJ, Chang YC. Immunohistochemical localization of tissue-type plasminogen activator and type I plasminogen activator inhibitor in radicular cysts. J Oral Pathol Med. 2004;33:156-61.

34- van Hinsbergh VW, Engelse MA, Quax PH. Pericellular proteolysis in angiogenesis and vasculogenesis. Arterioscler Thromb Vasc Biol. 2006;26:716-28.

35- Vaquero J, Zurita M, Oya S, Coca S, Salas C. Vascular permeability factor expression in cerebellar hemangioblastomas: correlation with tumor-associated cysts. J Neurooncol. 1999;41:37.

36- Walker KF, Lappin DF, Takahashi K, Hope J, Macdonald DG, Kinane DF. Cytokine expression in periapical granulation tissue as assessed by immunohistochemistry. Eur J Oral Sci. 2000;108:195201. 